\title{
Solving High-Speed Rail Planning with the Simulated Annealing Algorithm
}

By Ana Laura Costa $^{(1)}$, Maria da Conceição Cunha ${ }^{(2)}$, Paulo A. L. F. Coelho ${ }^{(3)}$ and Herbert H. Einstein, F. ASCE ${ }^{(4)}$

\section{ABSTRACT}

High-speed rail (HSR) networks require large investments, and the performance of the infrastructure is affected by varying local environments, while subject to tight layout restrictions. This paper presents a fully-integrated 3D model to optimize the HSR alignment at a planning scale, which sets boundaries for the final project design. The model considers mandatory and desirable specifics for the locations to link and the geometry in both the plan view and the longitudinal profile. It also allows one to define prohibited and restricted land-use areas. A computational tool has been developed that takes into account the problem specifics using a Simulated Annealing Algorithm (SAA) to optimize the problem solution. The capabilities of the model and the tool are demonstrated with the application to an intentionally simple and synthetic case study, considering construction costs and problem constraints, for which sound results are obtained. Both the model and the tool can be expanded to incorporate additional complexity, establishing the basis for real applications and for further integration of geotechnical and hydrological risk factors that affect the HSR performance.

Subject Headings: High-speed rail; Planning; Optimization; Decision support systems

(1) PhD Student, Department of Civil Engineering, University of Coimbra, Rua Luís Reis Santos, Polo II, 3030-788, Coimbra, Portugal.

(2) Professor, Department of Civil Engineering, University of Coimbra, Rua Luís Reis Santos, Polo II, 3030-788, Coimbra, Portugal.

(3) Assistant Professor, Department of Civil Engineering, University of Coimbra, Rua Luís Reis Santos, Polo II, 3030788, Coimbra, Portugal.

(4) Professor, Department of Civil and Environmental Engineering, Massachusetts Institute of Technology, 77 Massachusetts Ave., Cambridge, MA 02139-4307 


\section{INTRODUCTION}

Worldwide, $8838 \mathrm{~km}$ of High-Speed Rail (HSR) lines are under construction and $16318 \mathrm{~km}$ are planned, together with existing lines, adding up to $42322 \mathrm{~km}$ for the year of 2025 (UIC 2011). While different configurations of HSR can fulfill the scope of a project, the chosen corridors and cross sections affect the construction and operation costs of the network, the quality of the service and the broader social, economic and environmental impacts. Especially considering the large public investments required, the HSR network configuration should be optimized in the planning stage, at which the macro location is defined that sets boundaries for the final project design.

Planning for HSR needs to cope with varying local environments and, among these, geotechnical and hydrological factors that affect the construction costs and the quality of the performance. Extreme events, such as storms, floods and earthquakes, have the ability to damage linear transportation systems with important economic and social consequences (EQECAT 2002; Gordon et al. 1998; JGS 2006; Link 2010; USDOT 2002). The geotechnical and hydrological factors are location dependent and thus both the corridors and the cross-sections adopted can avoid or exacerbate an underperformance of the infrastructure. Also, the HSR layout is subject to tight restrictions, and safety and riding comfort require that only small tolerances are allowed (EC 2008). Standards and guidelines define minimum or maximum design values and consider a tolerance threshold up to a feasibility limit (EC 2008; UIC 2001).

This paper presents a fully integrated 3D model to optimize the HSR alignment at a planning scale, while complying with existing constraints. These include tight geometric allowances, land-use restrictions and desirable locations for the network. A computational tool has been developed (using Microsoft ® Visual Studio® C\# 2010 Professional linked to an SQL Server 2008 database) that implements the Simulated Annealing Algorithm (SAA) (Kirkpatrick et al. 1983) to solve the problem and optimize the problem solution. The sheer size of the problem dictates the use of a heuristic method (Murray and Church 1996).

The tool is applied to a synthetic case-study for standard planning conditions. Standard planning conditions refer to a scenario concerned with construction costs and problem constraints. The influence of the problem specifics and of the SAA implementation on the quality of the solutions are studied for a simple and small sized problem, in which these issues can be addressed, and an estimation of the SAA parameter combination that produces the best results is discussed. This systematic development of the tool is required in order to deal with the additional complexity inherent to real world problems and to the integration of geotechnical and hydrological risk factors.

\section{PREVIOUS RESEARCH IN THIS FIELD}

Much has been done regarding the optimization of railway infrastructure layout: Jha et al. (2007) propose an approach for optimizing transit rail lines when station locations are known and present an application to a case study with a $3.43 \mathrm{~km}$ distance between two stations. Samanta and Jha (2011) further determine the optimal location of rail stations 
to be connected by optimal alignments. The research by Jha et al. (2007) is based on previous works for highway alignment optimization. Similarities exist between the railway- and highway alignment optimization problems and comprehensive research exists for highways. Jong (1998) addresses the optimization of three-dimensional highway alignments using Genetic Algorithms (GA). This research was later extended with the incorporation of Geographic Information Systems (GIS) by Jha and Schonfeld (2000). Jong et al. (2000) propose a method for horizontal alignment optimization of highways between two given points, considering location dependent costs through a GIS database, and Fwa et al. (2002) address the optimization of the highway vertical alignment. Kim et al. (2005) develop a stepwise alignment optimization with GA for exploring computational burden issues and improving computational efficiency. Other specifics common to railway- and highway projects have been studied: Jha (2003) discusses environmental impacts and the selection of alignments along different corridors; Cheng and Lee (2006) propose a 3D alignment optimization considering heavy vehicle speeds; Kim et al. (2007) incorporate bridges and tunnels; Lee et al. (2009) address the horizontal alignment optimization problem considering areas where crossing is restricted and controlled and Kang et al. (2012) propose a highway alignment model that incorporates transition curves in the horizontal alignment and is able to deal with large data sets. The aforementioned research deals mainly with the alignment optimization for detailed stages of the project.

With a different perspective, Gipps et al. (2001) propose a transport route optimization planning tool using stochastic optimization techniques and consider, as stopping criterion, reaching the point when the spatial range of the possible alignments is such that a designer can work without concerns for the route's macro location. Also for the highway planning stage, Angulo et al. (2012) present a demand-based model for determining potential corridors.

The research overviewed, while extensive, does not comprise an integrated approach considering HSR specifics with a full 3D alignment optimization capable of analyzing large search space areas for a planning stage and expandable to incorporate geotechnical and hydrologic risks, which is addressed in this paper.

\section{MODEL FORMULATION}

The goal of the optimization problem is to find the HSR alignment configuration that minimizes an objective function considering construction costs while complying with demanding geometry, land-use and location issues. The 3D configuration of the HSR is, in reality, defined by a set of tangents and curves, both in the horizontal and vertical planes. The proposed model considers that the configuration is defined by linear sections that connect a set of sequential 3D points in space. The objective function, constraints and cost computation are discussed in the following sub-sections.

\section{OBJECTIVE FUNCTION}

The objective function, for a scenario of standard planning conditions, consists of the minimization of total costs given by the sum of five terms (Eq.1): construction costs $\Sigma_{(i, j) \in \Omega_{E}} C_{i j}$; penalty value for gradient noncompliance 
$\Sigma_{(i, j) \in \Omega_{N}} P_{\eta_{(i, j)}}$; penalty value for horizontal angle noncompliance $\Sigma_{(i, j, k) \in \Omega_{N}} P_{\beta_{(i, j, k)}}$; penalty value for land use noncompliance $\Sigma_{(i, j) \in \Omega_{N}} \Sigma_{s \in \Omega_{E}} P_{\lambda_{s}}$ and a location benefit term $\sum_{i \in \Omega_{N}} P_{v_{i}}$. The latter reflects a trade-off between additional construction or operational costs and linking cities or regions identified by the decision-maker as an added value to the network, for economic or equity reasons. Such locations are not fundamental for the scope of the project, otherwise the inclusion would be mandatory. Two levels of discretization are required: one related to the HSR configuration defining the set $\Omega_{N}$ of possible 3D nodes to be connected by the linear sections forming the network and another, discretizing the input mapped properties by defining the set $\Omega_{E}$ that subdivides the input maps into space property elements. Different map layers are used to characterize the space-search area (for example expropriation cost, land-use) and each layer map is subdivided into geo-referenced cells or elements $\left(\Omega_{E}\right)$ representing areas of constant properties, a rasterization process with an adequate size for the HSR planning. The five cost components in the objective function (Eq.1) are described in more detail below. The penalty and benefit coefficients should be established through expert judgment after consulting stakeholder panels and taking into consideration the problem specifics.

$$
\begin{aligned}
& \operatorname{Min} \sum_{(i, j) \in \Omega_{N}} C_{i j}\left[E x_{(i, j)}, E w_{(i, j)}, B_{(i, j)}, T_{(i, j)}\right]+\sum_{(i, j) \in \Omega_{N}} P_{\eta_{(i, j)}}\left[\eta_{(i, j)}, \eta_{\text {normal }}, \eta_{\lim i t}, \gamma_{\eta}\right]+ \\
& \sum_{(i, j, k) \in \Omega_{N}} P_{\beta_{(i, j, k)}}\left[\beta_{(i, j, k)}, \beta_{\text {normal }}, \beta_{\text {limit }}, \gamma_{\beta}\right]+\sum_{(i, j)=\Omega_{N}} \sum_{s \in \Omega_{E}} P_{\lambda_{s}}\left[l_{s}, \gamma_{\lambda_{s}}\right]-\sum_{i \in \Omega_{N}} P_{v_{i}}\left[\gamma_{v_{i}}\right]
\end{aligned}
$$

Where,

$\sum_{(i, j) \in \Omega_{E}} C_{i j}$ expresses the total construction costs and depends on the expropriation costs $E x_{(i, j)}$ and the costs of earthworks $E w_{(i, j)}$, bridges $B_{(i, j)}$ and tunnels $T_{(i, j)}$ of a linear section linking nodes $i$ and $j$;

$\Sigma_{(i, j) \in \Omega_{N}} P_{\eta_{(i, j)}}$ expresses the total penalty value for geometry gradient violation of each linear section linking nodes $i$ and $j$; it depends on the gradient value of the section $\eta_{(i, j)}$ and three problem parameters defining the normal gradient $\eta_{\text {normal }}$, the limit gradient $\eta_{\text {limit }}$ and the gradient penalty coefficient $\gamma_{\eta}$;

$\Sigma_{(i, j, k) \in \Theta_{N}} P_{\beta_{(i, j, k)}}$ expresses the total penalty value for geometry horizontal angle violation at each intermediate node $j$ of the configuration, formed by the two linear sections linking nodes $i, j$ and $k$; it depends on the angle value at node $j, \beta_{(i, j, k)}$, and three problem parameters defining the horizontal angle normal value $\beta_{\text {normal }}$, the horizontal angle limit value $\beta_{\text {limit }}$ and the horizontal angle penalty coefficient $\gamma_{\beta}$;

$\Sigma_{(i, j) \in \Omega_{N}} \sum_{s \in \Omega_{E}} P_{\lambda_{S}}$ expresses the total land use penalty value and depends on each space property element $s$ of restricted land use, length $l_{s}$ and land use penalty coefficient $\gamma_{\lambda s}$ in $\Omega_{E}$ that is overlaid by a linear section linking nodes $i$ and $j$; $\Sigma_{i \in \Omega_{N}} P_{v_{i}}$ expresses the total location benefit value and depends on the location coefficient $\gamma_{v i}$ of each node $i$ of $\Omega_{N}$. 


\section{CONSTRUCTION RELATED COST}

The construction $\operatorname{cost} C_{i j}$ of a linear section of the infrastructure linking nodes $i$ and $j$ is obtained by summing the expropriation costs $E x_{(i, j)}$, the construction costs of earthworks $E w_{(i, j)}$ (cuts and embankments), bridges $B_{(i, j)}$ and tunnels $T_{(i, j)}$ of that linear section. Track, catenary and signaling costs do not vary significantly with the in situ characteristics; as such they do not add major complexities to the model. However, the incorporation of existing length dependent costs favors shorter configurations and should be included in future real world applications.

The expropriation value $E x_{(i, j)}$ is obtained by overlaying, in plan view, the solution with a map of space property elements defining the unit cost per square meter. The total area to expropriate is established through an offset beyond the footprint of the infrastructure, as described in Costa et al. (2010). The total expropriation cost is then computed by summing, for all the space property elements within the area to expropriate, the element unit cost times its respective area.

The cross-section to adopt in each case depends on the difference between the ground and the HSR elevation and on the local ground conditions. The construction of bridges and tunnels is defined by thresholds of height and depth of the HSR in relation to the ground elevation. The costs of bridges and tunnels are assessed considering a linear relation to length and, for the latter, also depending on the local ground conditions. For cross-sections with height and depth below the bridge and tunnel thresholds, embankments and cuts are implemented. The cost computation of embankments and cuts earthworks, $E w_{(i, j)}$, requires the determination of the total volumes of excavation, embankment, sub-ballast, capping layer and ground improvement along each HSR linear section and the establishment of a unit cost for each of the items. The average-end area method discussed by Hintz and Vonderohe (2011) is used for the volume calculation of earthworks, as presented by Costa et al. (2010). The method basically consists in averaging the area measured in two consecutive cross-sections $A_{c}$ and $A_{c+l}$ and multiplying it by the distance $d$ between the two. At each cross-section, the slopes of cuts and embankments and the thicknesses of sub-ballast, capping and ground improvement depend on the ground behavior, which can be obtained by overlaying the HSR configuration with a ground behavior layer map. The requirement of different excavation methods (mechanical or blasting) also depends on the local ground conditions and affects the excavation unit cost.

\section{GEOMETRY PENALTY}

As previously discussed, design parameters in a HSR configuration have desirable values and allowances that can ascend up to mandatory limits. The consideration of penalties represents the trade-off between adopting the more restrictive desirable values and the additional costs that they may cause. The normal gradient $\eta_{\text {normal }}$ and the normal horizontal angle $\beta_{\text {normal }}$ specify the desirable design values that improve, amongst others, the energy consumption, braking distances, line speed and passenger comfort while decreasing forces on the track and the risk of derailment. Note 
that the use of limiting angles to derive the penalty is caused by the use of linear sections to represent the alignment Analogous penalties could be computed for circular and transition curves if such information is available. Geometry penalties, applied when normal values are not complied with, are defined by (Eq.2) and (Eq.3), respectively, for the gradient (Fig. 1) and the horizontal angle at intermediate nodes of the configuration. (For graphics describing the horizontal angle penalty formulation, which is analogous to the gradient formulation, refer to Costa et al. (2010).)

$$
\begin{aligned}
& P_{\eta_{(i, j)}}=\frac{\left|\eta_{\text {normal }}-\eta_{(i, j)}\right|}{\left|\eta_{\text {normal }}-\eta_{\lim i t}\right|} \gamma_{\eta} \\
& P_{\beta_{(i, j, k)}}=\frac{\left|\beta_{\text {normal }}-\beta_{(i, j, k)}\right|}{\left|\beta_{\text {normal }}-\beta_{\text {lim } i t}\right|} \gamma_{\beta}
\end{aligned}
$$

Where $P_{\eta_{(i, j)}}$ is the gradient penalty function of the linear section linking nodes $i$ and $j, \eta_{(i, j)}$ is the actual gradient of the linear section linking nodes $i$ and $j, \eta_{\text {normal }}$ and $\eta_{\text {limit }}$ are the normal and limit gradient values, $\gamma_{\eta}$ is the gradient penalty coefficient, $P_{\beta_{(i, j, k)}}$ is the horizontal angle penalty function at each intermediate node $j$, formed by the linear sections linking nodes $i, j$ and $k, \beta_{(i, j, k)}$ is the actual horizontal angle at the intermediate node $j, \beta_{\text {normal }}$ and $\beta_{\text {limit }}$ are the normal and limit values of the horizontal angle and $\gamma_{\beta}$ is the horizontal angle penalty coefficient.

\section{LAND USE PENALTY}

Land-use can be restricted in some areas of the territory for different reasons, including environmental and political decisions. The establishment of such areas intends to minimize the impacts on the existing natural or man-made environment by restricting the construction of new infrastructure. The decision maker can identify these as regions to avoid, provided that a trade-off with the additional costs is considered. A land use penalty (Eq.4) discourages configurations that cross land-use restricted areas, assessed by a unit length cost attributed to each of the space property element of $\Omega_{E}$ having restricted land-use. An overlay of the configuration linear sections on the land-use map layer identifies the restricted land use elements crossed by the HSR.

$$
P_{\lambda_{s}}=l_{s} \gamma_{\lambda_{s}}
$$

Where $P_{\lambda_{s}}$ is the land-use penalty of crossing the space property element $s$ of restricted land-use in $\Omega_{E}, l_{s}$ is the length of the space property element $s$ and $\gamma_{\lambda_{s}}$ is land use penalty coefficient of the space property element $s$.

\section{LOCATION BENEFITS}

The locations to connect with the HSR network are defined by the scope of the project. The decision makers define which cities or regions have to be served mandatorily by the network in order to fulfill the project's objective. However, serving additional cities and regions can be desirable for economic or equity purposes. Improved mobility and accessibility or political judgment can support the identified locations. However, trade-offs between accessing these 
locations and the additional capital- or operational costs need to be considered. This is accomplished by identifying nodes representing these locations and subtracting a benefiting term (Eq.5) that reduces the value of the objective function of HSR networks whose configurations include the respective nodes.

$$
P_{v_{i}}=\gamma_{v_{i}}
$$

Where $P_{v_{i}}$ is the location benefit of node $i$ in $\Omega_{N}$ that composes the HSR configuration and $\gamma_{v_{i}}$ is the location benefit coefficient of node $i$ in $\Omega_{N}$.

\section{Problem CONSTRAINTS}

The problem constraints define the search space for the problem and, depending on the constraints' specifics, difficulties to the search can be imposed but should be overcome by the algorithm implementation. The definition of a HSR configuration is constrained by the locations it is required to link. Additionally, track layout specifics and environmental issues affect the feasibility of the configuration. The proposed model considers three main constraint categories that influence the HSR configuration at a planning scale: location, geometry and land-use.

\section{LOCATION CONSTRAINT}

The location constraint ensures that the HSR network links all the mandatory locations established by the decision-maker. The constraint guarantees that all the nodes in $\Omega_{M N}$ are connected by the linear sections.

\section{GEOMETRY CONSTRAINTS}

Considering that codes exist and specify maximum limit gradients and minimum horizontal curvature radii (UIC 2001; EC 2008), two geometry constraints are formulated. The gradient constraint ensures that the actual gradient of each linear section $\eta_{(i, j)}$, either rising or falling, is smaller than the limit gradient $\eta_{\text {limit }}$. The simplification of defining the alignment by linear sections leads to the consideration of limit values for angles between sections $\beta_{\text {limit }}$, at intermediate nodes, instead of radii of curvature. The horizontal angle constraint ensures that all angles at intermediate nodes of the HSR configuration $\beta_{(i, j, k)}$ are larger than the limit value $\beta_{\text {limit }}$.

\section{LAND-USE CONSTRAINT}

The land-use constraint ensures that protected areas, where construction is prohibited, which are identified by forbidden land-use space property elements $\left(\Omega_{F E}\right)$ are not crossed by the HSR configuration. This is achieved by guaranteeing that the plan views of the linear sections forming the HSR configuration do not overlay any of the space property elements in $\Omega_{F E}$.

\section{THE SIMULATED ANNEALING ALGORITHM}

\section{OVerview}

The Simulated Annealing Algorithm is credited to Kirkpatrick et al. (1983) and traces its origins to the annealing process of materials to low energy states. In this physical process, the material is first heated, providing the energy 
necessary for particles to move, followed by a controlled cooling. Despite the fact that lower energy states relate to lower temperatures of the system, just a low temperature by itself is not sufficient (De Weck, Unpublished Memorandum, 2004). Slow cooling is necessary in order to allow particles to rearrange into the lowest energy configuration without being trapped in local minima energy states. The Metropolis algorithm (Metropolis et al. 1953) expresses this concept. Consider a current state $i$ of corresponding energy $E_{i}$. If state $i$ is perturbed into state $j$ of corresponding energy $E_{j}, j$ will be the new current state with a probability $p$ given by (eq.6):

$p=\min \left\{1, e^{\left(\frac{E_{i}-E_{j}}{k_{B} \cdot t}\right)}\right\}$

Where $k_{B}$ is the Boltzman's constant and $t$ is the temperature.

The application of the Metropolis criterion allows the SAA to accept worsening solutions and escape from local minima. The SAA changes solutions in a neighborhood defined in the vicinity of the current solution. Thus, when a current solution is a local minimum, attaining the global minimum requires that worse solutions are accepted. The rate of acceptance of worse solutions is governed by a cooling schedule and decreases as the SAA implementation progresses. For a comprehensive discussion of the SAA refer to Van Laarhoven and Aarts (1987).

\section{ALGORITHM IMPLEMENTATION}

Having established the model, two additional elements are needed for the SAA implementation: a procedure to generate candidate configurations and a cooling schedule.

The procedure to generate new candidate configurations establishes the neighborhood of any current configuration. The new candidate configuration is obtained by changing, randomly in the neighborhood, the current solution. The methodology adopted allows the 3D change of the configurations, by a random 3D repositioning of the nodes, within the neighborhood structure.

Different temperature decrease rates (cooling schedule) can be implemented (De Weck, Unpublished Memorandum, 2004). The choice relies on an exponential cooling schedule that allows the SAA to spend more time at lower temperatures. This implementation follows closely Cunha (1999) and Cunha and Sousa (2001) that present, respectively, flowcharts for the SAA implementation and the cooling schedule and the pseudo-code for the SAA implementation. Four parameters are considered in the cooling schedule:

- $a$ : the elasticity of acceptance that defines the probability of accepting a transition from the initial configuration to a new candidate configuration that yields higher cost than the initial one. The probability is defined as a percentage and it is used to define the initial temperature of the algorithm by $t_{1}=-0.1 c\left(s_{1}\right) / \ln (a)$, where $c\left(s_{1}\right)$ is the cost of the initial 
configuration. This expression defines an initial temperature that allows $a \%$ of configurations with a $10 \%$ higher cost than the initial configuration to be accepted.

- $n_{l}$ : the minimum number of algorithm iterations to be performed at each temperature, even without improvement of the optimum or the average cost. Equilibrium is to be reached at each temperature, meaning that no overall best configuration is attained or the average cost of the evaluated configurations does not improve.

- $r$ : the rate of temperature decrease, whenever a temperature decrease occurs. An exponential decrease rate is chosen with a constant $r$ factor. The temperature decrease at each level is governed by $t_{k}=r^{k} t_{l}$. According to De Weck (Unpublished Memorandum, 2004), the value chosen is of great influence on the quality of the results achieved: if the rate is too low (large $r$ ) the algorithm performance can resemble a random search (Solis and Wets 1981), whereas small values of $r$ can relate to a gradient descent behavior (Griewank 1981) and/or a premature termination of the algorithm. - $n_{2}$ : the number of temperature decreases to be performed without improvement of the optimum or the average cost. It establishes the stopping criterion of the algorithm.

\section{SYNTHETIC CASE STUDY}

The model has been applied to a synthetic case study and solved with the SAA, following the guidelines in the previous section. Standard planning conditions are considered, meaning that a scenario concerned with construction costs and problem constrains is addressed. Additional applications were run for performing a sensitivity analysis on the geometry, land use and location coefficients. The following sub-sections present the characteristics and the results obtained for the base case and the sensitivity analysis.

The SAA may be applied to a wide range of problems (Dekkers and Aarts 1991), although the number of iterations necessary to reach equilibrium at each temperature, for achieving optimal solution, is exponentially dependent on problem-size (Aarts and Vanlaarhoven 1985). Solving a large problem such as the HSR network planning problem becomes unreasonable with an exponential complexity dependent on the number of iterations and thus the implementation of the algorithm includes a study for the estimation as to which combination of the SAA parameters works best.

\section{Characteristics OF THE BaSe CaSe}

The synthetic problem aims at linking three mandatory locations with a HSR network in a rectangular shaped area of $60 \mathrm{~km}$ per $40 \mathrm{~km}$ (Fig. 2(a)), discretized by the 3D mesh in Fig. 2(b) that spans $2 \mathrm{~km}$ in each plan view direction and 10 $\mathrm{m}$ vertically and defines the permissible node positions $\left(\Omega_{N}\right)$. The mandatory locations, defined by their $(\mathrm{x}, \mathrm{y})$ coordinates, are $\mathrm{M} 1$ at $(0 \mathrm{~km}, 0 \mathrm{~km}), \mathrm{M} 2$ at $(40 \mathrm{~km}, 20 \mathrm{~km})$ and $\mathrm{M} 3$ at $(58 \mathrm{~km}, 38 \mathrm{~km})$. An additional desirable location B is considered at $(30 \mathrm{~km}, 4 \mathrm{~km})$ with a location benefit term to be subtracted to the objective function value when the node is connected by the HSR linear sections, with $\gamma_{v_{B}}=30 \% \Sigma_{(i, j) \in \Omega_{N}} C_{i j}$. Four input spatial properties are mapped and represented by 
different raster data type layers: elevation, ground behavior, expropriation cost and land use type. The layers are discretized in $200 \mathrm{~m}$ wide square space property elements $\left(\Omega_{E}\right)$ that establish a constant value of elevation, ground behavior, expropriation cost and land-use within the boundaries of each element. Penalties to optimize the geometric and land use design are proportional to the construction cost of the configuration and consider the following coefficients: $\gamma_{\eta}=5 \% \Sigma_{(i, j) \in \Omega_{N}} C_{i j}$ for gradient, $\gamma_{\beta}=5 \% \Sigma_{(i, j) \in \Omega_{N}} C_{i j}$ for horizontal angle and $\gamma_{\lambda_{s}}=2 \% \Sigma_{(i, j) \in \Omega_{N}} C_{i j}$ for land use. The normal and limit gradients and horizontal angles are $\eta_{\text {normal }}=20 \%, \eta_{\text {limit }}=35 \%$, $\beta_{\text {limit }}=100^{\circ}$ and $\beta_{\text {normal }}=120^{\circ}$. Fig. 2 (a) identifies the forbidden land-use elements, the restricted land-use elements that, if crossed by the HSR, incur a land use penalty and the elements exempt from restrictions. The cross-sections, for earthworks and cost calculation, are evaluated every $200 \mathrm{~m}$ measured along the longitudinal profile of the configuration.

\section{RESULTS OF THE BASE CASE}

The SAA was applied to solve the model considering 15 random seed numbers for combinations of SAA parameters varying the elasticity of acceptance $a(0.8,0.9,0.93)$, the temperature decrease rate $r(0.7,0.8,0.9,0.93)$, the minimum number of computed solutions at each temperature $n_{1}(1000,1500,2000,5000,10000)$ and the termination criterion $n_{2}(5,10)$.

Computations revealed that increasing values of the elasticity of acceptance $a$ correspond, as expected, to an increasing percentage of accepted configurations at the initial temperature (Fig.3). Kirkpatrick (1984) and Van Laarhoven and Aarts (1987) suggest that the initial temperature should be such that at least $80 \%$ of computed configurations are accepted at that temperature, a condition that exists for all tested values of $a$. Additionally, initial SAA runs performed indicate that a minimum number of iterations $n_{1}=5000$ and a stopping criterion $n_{2}=10$ from, respectively, $(1000,1500,2000,5000,10000)$ and $(5,10)$, provide the least costly solutions for the different remaining SAA parameters (elasticity of acceptance $a$ and temperature decrease rate $r$ ).

Fig. 3 considers $n_{1}=5000$ and $n_{2}=10$ and shows the variation of the configuration cost (objective function value) average with the temperature decrease rate $r$, for different values of elasticity of acceptance $a$. Each point in Fig. 3 represents the average cost of the configurations obtained from solving the model with 15 runs (one for each random seed number) for that same SAA parameter set. One observes that the average cost tends to decrease with increasing $r$ up to 0.9. A larger rate $(r=0.93)$ results, on average, in equal or costlier solutions than those obtained for $r=0.9$.

20 additional SAA runs were performed considering $a=0.9, r=0.9, n_{1}=5000$ and $n_{2}=10$, the SAA parameter set for which the lowest average cost is obtained (Fig. 3). The same overall best configuration was found and only few SAA runs converged for different solutions, with a cost up to 5\% larger, as one could expect from a random search algorithm such as the SAA. Fig. 4 shows the convergence history, evolution of the last accepted configuration before a temperature 
decrease and of the current optimum, of one SAA run for $a=0.9, r=0.9, n_{1}=5000$ and $n_{2}=10$. Large objective function value configurations are accepted in the early temperature stages of the implementation, allowing for a comprehensive exploration of the problem search space. As the algorithm progresses the acceptance of worsening solutions decreases and convergence to the best overall configuration occurs.

Tables 1 and 2 present, respectively, the cost breakdown and the geometry of the best overall configuration found for the base case; Figs. 5(a) and (b) present the configuration plan view overlaying, respectively, the elevation layer and the land-use layer. The configuration cost (objective function value) is $73,235,813 €$ and is the result of the construction costs and the location benefit: the geometry penalty is null since the gradient of each linear section and the angle at each intermediate node are, respectively, smaller and larger than, respectively, the normal values $\eta_{\text {normal }}=20 \%$ and $\beta_{\text {normal }}=120^{\circ}$; the land-use penalty is also null since the configuration does not cross restricted land-use elements (Fig. 5(b)). The location benefit term, however, is not null as the desirable location B at $(30 \mathrm{~km}, 4 \mathrm{~km})$ is linked by the HSR configuration. The configuration also avoids sharp variations of the ground elevation (Fig. 5(a)), produces a vertical adjustment of the longitudinal profile that does not require the construction of bridges and tunnels, costlier than embankments and cuts.

\section{SENSITIVITY ANALYSIS}

\section{PENALTY COEFFICIENTS}

For each penalty, SAA runs were made changing only the respective coefficient value (gradient $\gamma_{\eta}$, horizontal angle $\gamma_{\beta}$ and land-use $\gamma_{\lambda_{\mathrm{s}}}$ ) and maintaining all the remaining specifics from the base case. The best configuration found for the base case complies with the desirable normal values for geometry (Table 2) and does not cross restricted land use elements (Fig. 5(b)), thus the penalties were null (Table 1). To assess how smaller coefficients could lead to a solution with smaller objective function values, with a trade-off between the construction costs and the violation of the desirable restrictive values, the penalty coefficients were reduced to $1 / 5$ of their base case value. The new coefficient values tested are $\gamma_{\eta}=1 \% \Sigma_{(i, j) \in \Omega_{\mathrm{N}}} C_{i j}$ for gradient, $\gamma_{\beta}=1 \% \Sigma_{(i, j) \in \Omega_{\mathrm{N}}} C_{i j}$ for horizontal angle and $\gamma_{\lambda_{\mathrm{s}}}=0.4 \% \Sigma_{(i, j) \in \Omega_{\mathrm{N}}} C_{i j}$ for land use.

The best configuration found for each variation of the penalty coefficients is equal to the best configuration of the base case. This means that even for smaller values of the coefficients, tested separately, the best solution complies with the gradient and horizontal angle normal values and does not cross a restricted land use area.

\section{LOCATION BENEFIT}

The influence of the location benefit coefficient was studied by varying its value with two additional situations: $\gamma_{v_{\mathrm{B}}}=15 \% \sum_{(i, j) \in \Omega_{\mathrm{N}}} C_{i j}$ and $\gamma_{v_{\mathrm{B}}}=0$, keeping the penalty coefficients unchanged from the base case.

The solution found considering $\gamma_{v_{\mathrm{B}}}=15 \% \Sigma_{(i, j) \in \Omega_{\mathrm{N}}} C_{i j}$ has the same geometry as the base case solution (Table 2;

Fig. 5) and thus equal construction costs and penalty costs (Table 1). The objective function value, however, differs by 
the smaller location benefit: instead of benefiting the objective function by $31,386,777 €$ as in the base case, the new location benefiting term is half of this and the objective function value is $104,622,590-15,693,388=88,929,202 €$.

The best HSR configuration found for the null benefit coefficient $\gamma_{v B}=0$ is distinct from the previous one both in terms of cost (Table 1) and geometry (Table 2; Fig. 5(c);Fig. 5(d)). While the configuration is shorter and construction costs are $9 \%$ smaller than the base case solution, the objective function yields a larger value. This is due to the lack of the location benefit: earthworks and expropriation costs are smaller and penalties are equally null.

\section{NORMAL AND LIMIT HORIZONTAL ANGLES}

Considering that the best configuration found for the base case has 3 angles smaller than $140^{\circ}$, two of which are smaller than $130^{\circ}$, additional computations were performed for $\beta_{\text {limit }}=130^{\circ}$ and $\beta_{\text {normal }}=140^{\circ}$. The results obtained (Table 1; Table 2; Fig. 5 (e); Fig. 5 (f)) are similar to the base case but complying with the new and more restrictive normal and limit values. One can observe that the configuration still crosses the desirable node, and the objective function is improved by the location benefit and no other penalties are applied. In fact, the configuration changes slightly from the base case, producing all intermediate angles larger than $140^{\circ}$ at a larger construction cost than the base case.

\section{CONCLUSIONS}

A model for optimizing the preliminary design of high-speed rail networks has been presented. The model represents location dependent properties influencing the configuration, as well as considering the HSR design requirements and best practice design parameters. It aims at optimizing conflicting design choices that influence the performance of the infrastructure. The sheer size of the problem imposed the use of a heuristic method, a Simulated Annealing Algorithm (SAA), to solve the model.

A user-friendly tool has been developed that can consider the model input location data, the HSR 3D geometry restrictions and the SAA. The model's capabilities are demonstrated with the application to an intentionally simple and synthetic case study. A scenario of standard planning conditions, considering construction costs and problem constraints, has been used and the combination of SAA parameters that works best is presented. A sensitivity analysis has been performed, varying the model penalty and benefit coefficients and the horizontal angle normal and limit design values. Sound solutions have been obtained in all cases, revealing the capabilities of the model and the problem solving methodology in addressing the preliminary design optimization for HSR networks. Furthermore, the fact that the HSR configuration remains unchanged for the different penalty coefficients, changes slightly to include more restrictive horizontal angle values and has a reduced length for the null location benefit coefficient, suggests that the solution in this particular case is fairly robust to variations of the model parameters. Both the model and the tool presented in this paper can be expanded to incorporate additional complexity, establishing the basis for real world applications in which the integration of geotechnical and hydrological risk factors affecting the HSR performance can also be considered. 


\section{ACKNOWLEDGEMENTS}

The authors greatly acknowledge the generous support of the Government of Portugal through FCT grant (SFRH/BD/43012/2008) and the MIT-Portugal Program.

\section{NOTATION}

$1_{\mathrm{s}}$ - Length of space property element $s$.

$\beta_{(\mathrm{i}, \mathrm{j}, \mathrm{k})}$ - Angle measured in the horizontal projection of the configuration at each intermediate node $\mathrm{j}$ formed by the two linear sections linking nodes $\mathrm{i}$ and $\mathrm{j}$ and $\mathrm{j}$ and $\mathrm{k}$.

$\beta_{\text {limit }}$ - Horizontal angle limit value.

$\beta_{\text {normal }}$ - Horizontal angle normal value.

$\gamma_{\beta}$ - Horizontal angle penalty coefficient.

$\gamma_{\eta^{-}}$Gradient penalty coefficient.

$\gamma_{\lambda s}$ - Land use penalty coefficient of space property element $s$ contained in $\Omega_{E}$

$\gamma_{v i}$ - location benefit coefficient of node $i$ contained in $\Omega_{N}$

$\eta_{(\mathrm{i}, \mathrm{j})}-$ Gradient of linear section linking nodes $i$ and $j$.

$\eta_{\text {limit }}$ - Gradient limit value.

$\eta_{\text {normal }}$ - Gradient normal value.

$\Omega_{\mathrm{E}}-$ Set of all space property elements.

$\Omega_{\mathrm{FE}}$ - Subset of $\Omega_{\mathrm{E}}$ containing all space property elements of forbidden land-use.

$\Omega_{\mathrm{MN}}$ - Subset of $\Omega_{\mathrm{N}}$ containing all mandatory nodes.

$\Omega_{\mathrm{N}}$ - Set of all three-dimensional nodes of the discretization mesh.

\section{REFERENCES}

Aarts, E. H. L., and Vanlaarhoven, P. J. M. (1985). "Statistical cooling: a general approach to combinatorial optimization problems." Philips Journal of Research, 40(4), 193-226.

Angulo, E., Castillo, E., Garcia-Rodenas, R., and Sanchez-Vizcaino, J. (2012). "Determining highway corridors." Journal of Transportation Engineering, 138(5), 557-570.

Cheng, J. F., and Lee, Y. S. (2006). "Model for three-dimensional highway alignment.” Journal of Transportation Engineering, 132(12), 913-920.

Costa, A.L., Coelho, P., Cunha, M. and Einstein, H. (2010). "Tools for high-speed rail planning optimization: preliminary developments of a case study." General Proceedings of the 12th World Conference on Transport Research Society, Lisbon, Portugal.

Cunha, M. D. (1999). “On solving aquifer management problems with simulated annealing algorithms.” Water Resources Management, 13(3), 153-169. 
Cunha, M., and Sousa, J. (2001). "Hydraulic infrastructures design using simulated annealing." Journal of Infrastructure Systems, 7(1), 32-39.

Dekkers, A., and Aarts, E. (1991). “Global optimization and simulated annealing." Mathematical Programming, 50(1), 367-393.

EC (2008). "Commission Decision of 20 December 2007 concerning a technical specification for interoperability relating to the infrastructure sub-system of the trans-European high-speed rail system." Official Journal of the European Union, <http://eur-lex.europa.eu/> (Nov. 30, 2011).

EQECAT (2002). Central European Flooding, August 2002. Oakland, CA 94612, 1-21.

Fwa, T. F., Chan, W. T., and Sim, Y. P. (2002). “Optimal vertical alignment analysis for highway design.” Journal of Transportation Engineering, 128(5), 395-402.

Gipps, P. G., Gu, K. Q., Held, A., and Barnett, G. (2001). "New technologies for transport route selection." Transportation Research Part C: Emerging Technologies, 9(2), 135-154.

Gordon, P., Richardon, H. W., and Davis, B. (1998). “Transport-related impacts of the Northridge Earthquake.” Journal of Transportation and Statistics, 1(2), 21-36.

Griewank, A. O. (1981). "Generalized descent for global optimization.” Journal of Optimization Theory and Applications, 34(1), 11-39.

Hintz, C., and Vonderohe, A. P. (2011). “Comparison of Earthwork Computation Methods.” Transportation Research Record, 2215, 100-104.

JGS (2006). "Ground damage resulting from torrential rains in Fukui, July 2004.” Soils and Foundations, 46(6), 869884.

Jha, M. K. (2003). “Criteria-based decision support system for selecting highway alignments.” Journal of Transportation Engineering, 129(1), 33-41.

Jha, M. K., and Schonfeld, P. (2000). "Integrating genetic algorithms and geographic information system to optimize highway alignments." Transportation Research Record: Journal of the Transportation Research Board, Washington, D.C., 1719, 233-240.

Jha, M. K., Schonfeld, P., and Samanta, S. (2007). "Optimizing rail transit routes with genetic algorithms and geographic information system.” Journal of Urban Planning and Development, 133(3), 161-171.

Jong, J C, Jha, M. K., and Schonfeld, P. (2000). "Preliminary highway design with genetic algorithms and geographic information systems." Computer-Aided Civil and Infrastructure Engineering, 15(4), 261-271.

Jong, J. C. (1998). “Optimizing highway alignments with genetic algorithms.” PhD dissertation, Department of Civil and Environmental Engineering, University of Maryland, College Park, Md.

Kang, M. W., Jha, M. K., and Schonfeld, P. (2012). “Applicability of highway alignment optimization models.” Transportation Research Part C: Emerging Technologies, 21(1), 257-286.

Kim, E., Jha, M. K., Schonfeld, P., and Kim, H. S. (2007). "Highway alignment optimization incorporating bridges and tunnels." Journal of Transportation Engineering, 133(2), 71-81.

Kim, E., Jha, M. K., and Son, B. (2005). "Improving the computational efficiency of highway alignment optimization models through a stepwise genetic algorithms approach.” Transportation Research Part B: Methodological, 39(4), $339-360$. 
Kirkpatrick, S. (1984). "Optimization by simulated annealing: quantitative studies.” Journal of Statistical Physics, 34(56), 975-986.

Kirkpatrick, S., Gelatt, C. D., and Vecchi, M. P. (1983). “Optimization by simulated annealing.” Science, 220(4598), 671-680.

Lee, Y., Tsou, Y. R., and Liu, H. L. (2009). “Optimization method for highway horizontal alignment design.” Journal of Transportation Engineering, 135(4), 217-224.

Link, L. E. (2010). "The anatomy of a disaster, an overview of Hurricane Katrina and New Orleans." Ocean Engineering, $37(1), 4-12$.

Metropolis, N., Rosenbluth, A. W., Rosenbluth, M. N., Teller, A. H., and Teller, E. (1953). "Equation of State Calculations by Fast Computing Machines." Journal of Chemical Physics, 21(6), 1087-1092.

Murray, A. T., and Church, R. L. (1996). "Applying simulated annealing to location-planning models.” Journal of Heuristics, 2(1), 31-53.

Samanta, S., and Jha, M. K. (2011). "Modeling a rail transit alignment considering different objectives." Transportation Research Part A: Policy and Practice, 45(1), 31-45.

Solis, F. J., and Wets, R. J. B. (1981). "Minimization by random search techniques.” Mathematics of Operations Research, 6(1), 19-30.

UIC (2001). "Design of New Lines for Speeds of 300-350 Km/h, State of the Art." International Union of Railways, <http://www.uic.org/IMG/pdf/2-09_Repor350_en.pdf> (Dec.19, 2011).

UIC (2011). "High Speed Lines in the World.” International Union of Railways, <http://www.uic.org/spip.php?article573> (Dec.15, 2011).

USDOT (2002). Effects of Catastrophic Events on Transportation System Management and Operations, Northridge Earthquake-January 17, 1994. Washington, DC 20590.

Van Laarhoven, P. J. M., and Aarts, E. H. L. (1987). Simulated annealing: theory and applications. Kluwer Academic Publishers Group, Dordrecht, The Netherlands, 55-62. 
Table 1 - Cost breakdown of HSR configurations.

\begin{tabular}{|l|c|c|c|}
\hline & Base Case & $\boldsymbol{\gamma}_{\mathbf{v B}}=\mathbf{0}$ & $\boldsymbol{\beta}_{\text {normal }} / \boldsymbol{\beta}_{\text {limit }}$ \\
\hline Configuration Cost & $\mathbf{7 3 2 3 5 8 1 3}$ & $\mathbf{9 5 6 7 3 7 8 1}$ & $\mathbf{7 8 1 9 1 8 0 9}$ \\
\hline 1. Construction Cost & 104622590 & 95673781 & 111702584 \\
\hline 1.1 Expropriation & 75261796 & 71119151 & 78380116 \\
\hline 1.2 Earthworks & 29360794 & 24554630 & 33322468 \\
\hline 1.3 Bridges & 0 & 0 & 0 \\
\hline 1.4 Tunnels & 0 & 0 & 0 \\
\hline 2. Geometry Penalty & 0 & 0 & 0 \\
\hline 3. Land-Use Penalty & 0 & 0 & 0 \\
\hline 4. .Location Benefit & 31386777 & 0 & 33510775 \\
\hline
\end{tabular}

460 
Table 2 - Geometry of HSR configurations.

\begin{tabular}{|c|c|c|c|c|c|c|c|c|c|}
\hline & \multicolumn{4}{|c}{ Base Case } & \multicolumn{3}{c|}{$\boldsymbol{\gamma}_{\mathbf{v B}}=\mathbf{0}$} & \multicolumn{3}{c|}{$\boldsymbol{\beta}_{\text {normal }} / \boldsymbol{\beta}_{\text {limit }}$} \\
\hline & $(\mathbf{x}, \mathbf{y}, \mathbf{z})$ & $\boldsymbol{\eta}$ & $\boldsymbol{\beta}$ & $\mathbf{( x , y , z})$ & $\boldsymbol{\eta}$ & $\boldsymbol{\beta}$ & $(\mathbf{x}, \mathbf{y}, \mathbf{z})$ & $\boldsymbol{\eta}$ & $\boldsymbol{\beta}$ \\
\hline Node & $(\mathrm{km}, \mathrm{km}, \mathrm{m})$ & $(\%)$ & $\left(^{\mathbf{o}}\right)$ & $(\mathrm{km}, \mathrm{km}, \mathrm{m})$ & $(\%)$ & $\left(^{\mathbf{o}}\right)$ & $(\mathrm{km}, \mathrm{km}, \mathrm{m})$ & $(\% \mathrm{o})$ & $\left(^{\mathbf{o}}\right)$ \\
\hline 1 & $(0,0,10)$ & 1.58 & & $(0,0,10)$ & 0.00 & & $(0,0,10)$ & 0.79 & \\
\hline 2 & $(6,2,20)$ & 0.00 & 166 & $(8,8,10)$ & 1.58 & 153 & $(12,4,20)$ & 0.00 & 153 \\
\hline 3 & $(30,4,20)$ & 1.56 & 133 & $(26,14,40)$ & 0.00 & 177 & $(26,2,20)$ & 2.24 & 145 \\
\hline 4 & $(38,14,40)$ & -1.58 & 160 & $(36,18,40)$ & 2.24 & 175 & $(30,4,30)$ & 0 & 149 \\
\hline 5 & $(40,20,30)$ & -0.56 & 135 & $(40,20,30)$ & 0.56 & 180 & $(40,20,30)$ & 0 & 144 \\
\hline 6 & $(56,28,20)$ & 0.00 & 128 & $(56,28,20)$ & 0.00 & 128 & $(50,24,30)$ & -1.24 & 142 \\
\hline 7 & $(58,38,20)$ & & & $(58,38,20)$ & & & $58,38,20)$ & & \\
\hline
\end{tabular}




\section{Figure 1}

Click here to download Figure: Figure 1_final_w_captions.pdf

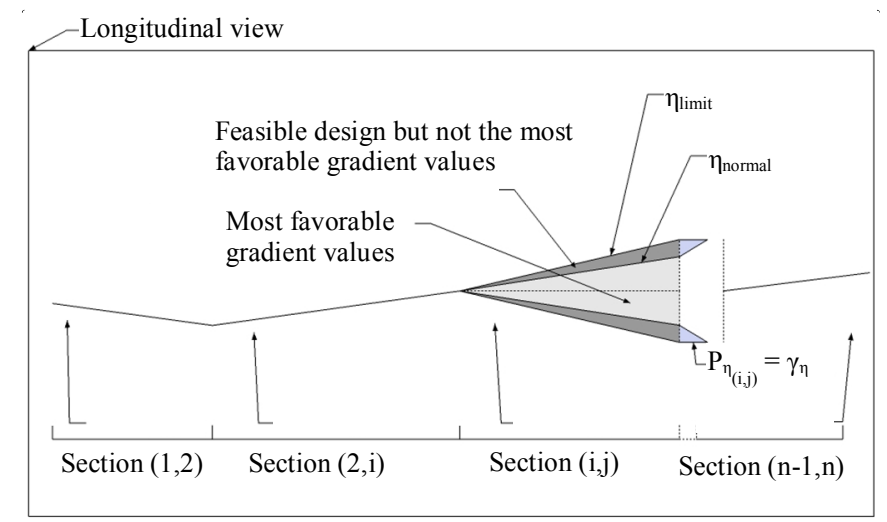

Figure 1 - Gradient penalty formulation . 


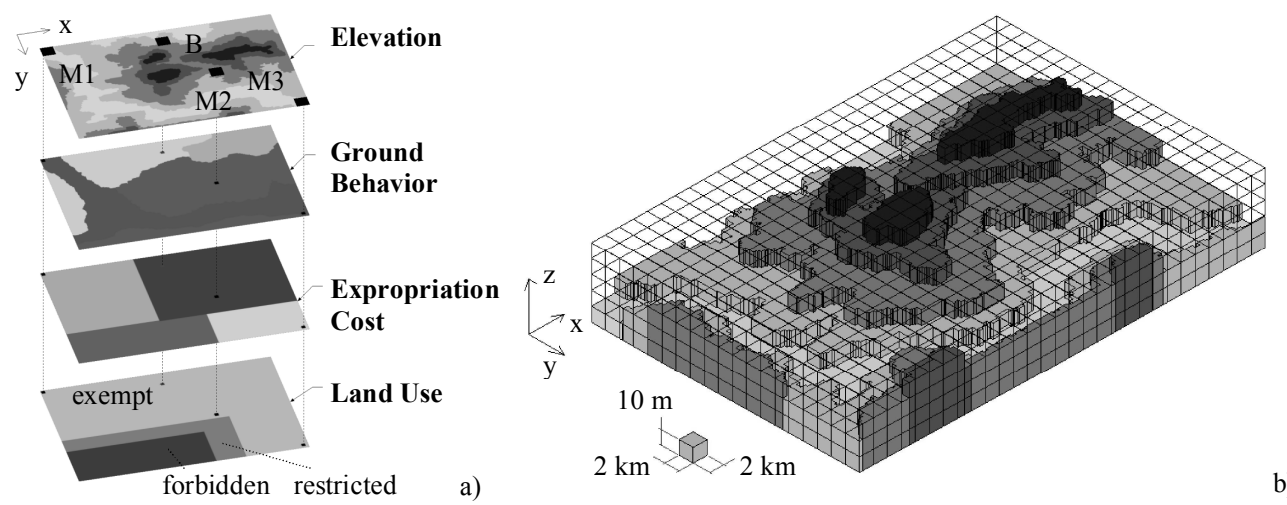

b)

1 Figure 2-Case study specifics: a) Nodes overlaying the elevation-, ground behavior-, expropriation cost - and land-use

2 layers; b) Ground elevation and 3D mesh defining the permissible node positions in each configuration. 


\section{Figure 3}

Click here to download Figure: Figure 3_final_w_captions.pdf

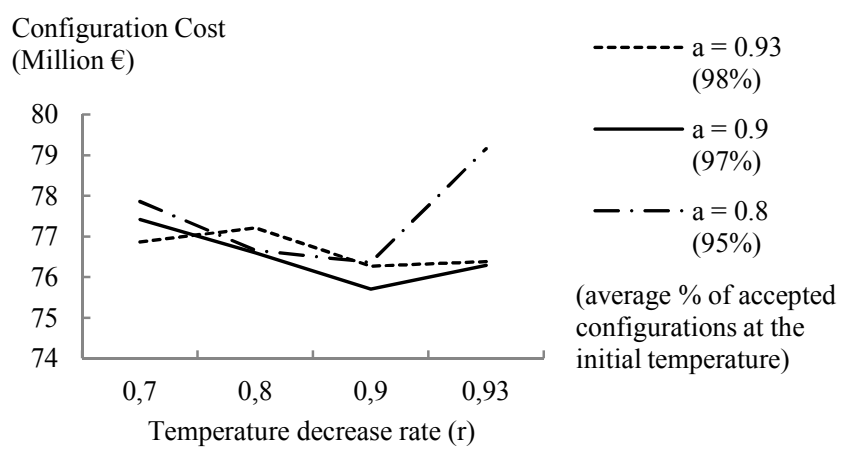

Figure 3 - Influence of temperature decrease rate $r$ on the average cost of the configurations. 


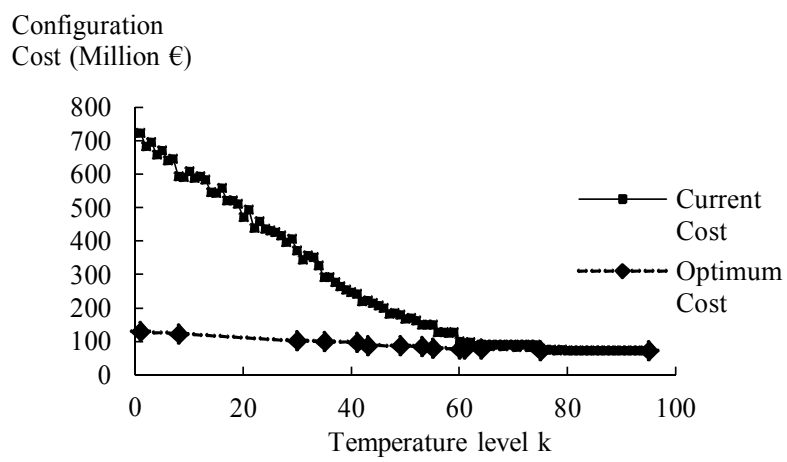

Figure 4 - Convergence history for $\mathrm{a}=0.9, \mathrm{r}=0.9, \mathrm{n} 1=5000$ and $\mathrm{n} 2=10$ : evolution of the last accepted configuration before a temperature decrease and of the current optimum at the time of each temperature decrease. 

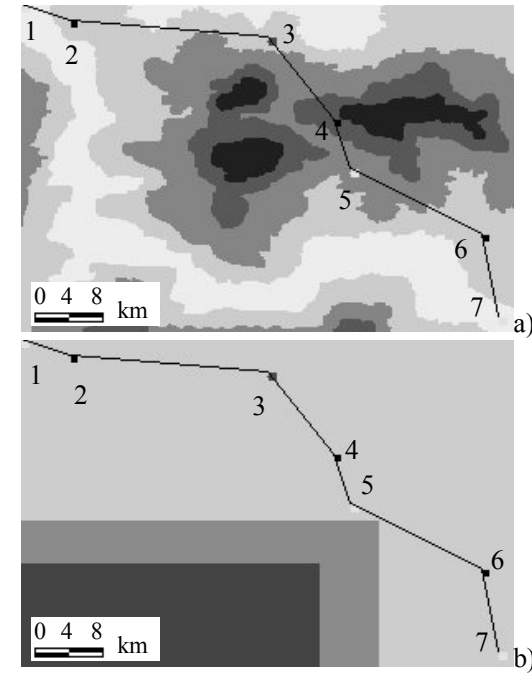

a)

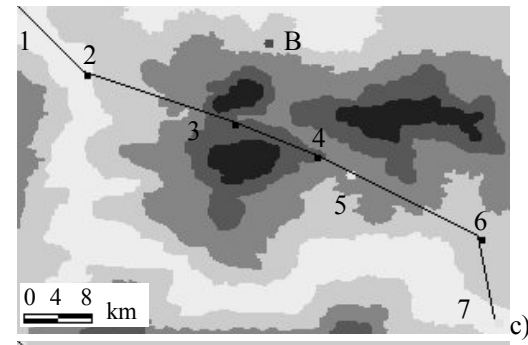

c)

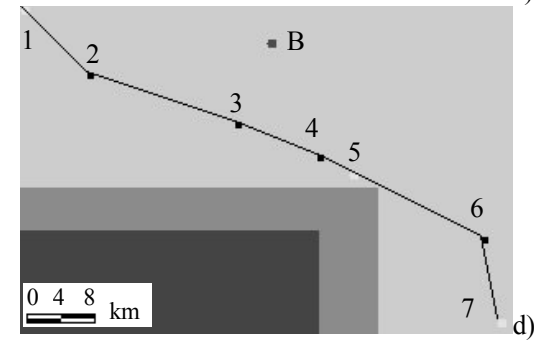

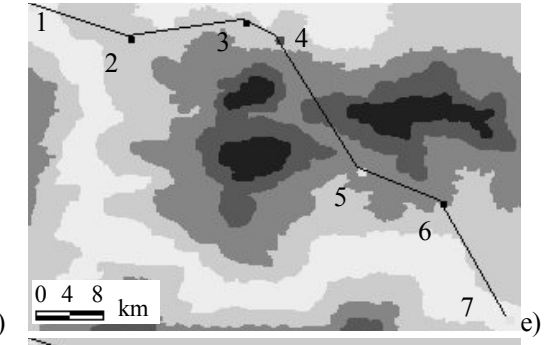

$\stackrel{04}{0} \mathrm{~km}$

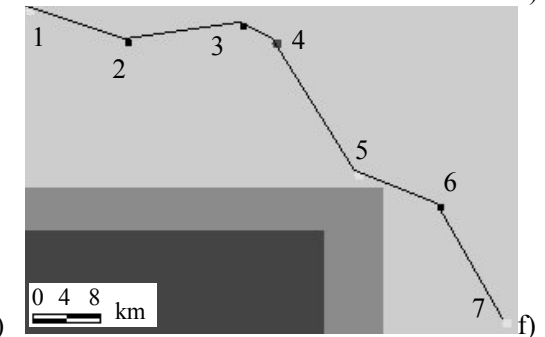

Figure 5 - Plan view of the best configurations: (a) and (b) for the base case, (c) and (d) for $\gamma_{v B}=0$ and (e) and (f) for sensitivity analysis of $\beta_{\text {normal }} / \beta_{\text {limit }}$; configurations overlay the ground elevation layer in (a), (c) and (e) and the land-use layer in (b), (d) and (f). 
Figure 1 - Gradient penalty formulation.

Figure 2- Case study specifics: a) Nodes overlaying the elevation-, ground behavior-, expropriation cost- and land-use layers; b) Ground elevation and 3D mesh defining the permissible node positions in each configuration.

Figure 3 - Influence of temperature decrease rate $r$ on the average cost of the configurations.

Figure $4-$ Convergence history for $\mathrm{a}=0.9, \mathrm{r}=0.9, \mathrm{n} 1=5000$ and $\mathrm{n} 2=10$ : evolution of the last accepted configuration before a temperature decrease and of the current optimum at the time of each temperature decrease.

Figure 5 - Plan view of the best configurations: (a) and (b) for the base case, (c) and (d) for $\gamma_{v B}=0$ and (e) and (f) for sensitivity analysis of $\beta_{\text {normal }} / \beta_{\text {limit }}$; configurations overlay the ground elevation layer in (a), (c) and (e) and the land-use layer in (b), (d) and (f). 